\title{
ATROPHIC RECESSION OF THE LAMINA CRIBROSA
}

BY

\section{A. Christie Reid}

NOTTINGHAM

MY apology for this paper is that several points in the pathogenesis of a condition that is not yet universally recognized or even acknowledged are still obscure.

The condition I have in view is one in which, without any demonstrable increase of tension and with the minimum of interference with the nerve elements passing through it the lamina is " receded," giving a hollowed-out appearance indistinguishable from glaucomatous cupping.

In the living subject this is accompanied by a wider exposure of the lamina of a measurable amount, as has been so well impressed on us by Mr. Ransom Pickard in his numerous papers.

That there may be such a condition apart from any increase of intra-ocular pressure should not be regarded as a physical impossibility. But on showing a case of this nature to a recent meeting I was faced with two curious objections (1) that the tissues of the optic nerve were absolutely incompressible, in the same way as any fluid is incompressible in a physical sense. This objection comes from a confusion of ideas. All tissues of the body are compressible because ultimately all are exposed to the compressible air that surrounds it. In other words we are not dealing with any tissue enclosed in an absolutely and permanently rigid envelope. All our so-called "rigid envelopes" can yield to pressure if time is given, because they are also living structures that can undergo changes in their structure and chemical composition. That this is true of the coats of the eyeball is proved by the quadrate eye of old untreated glaucoma.

(2) It was also objected that the pressures behind and in front of the lamina must be equal because we have there a state of equilibrium.

But the walls of a golf ball are in equilibrium with a centre pressure so much in excess that we know the risk of cutting into it. So with a tennis ball to a less degree. Equilibrium in these cases depends on three factors, the inside and outside pressures and the rigidity of the wall between them.

So with the eyeball; the rigidity of the wall is the factor that can maintain a position of equilibrium between the intra-ocular and the atmospheric or the tissue pressure, according to which is in contact with the part concerned.

In the case of the lamina it is the tissue pressure of the optic 
nerve behind it, though this is of course in indirect communication through the body with the outer air.

My contention in showing the case - a lady, aged 70 years, tension decidedly subnormal, 26 McLean, with obvious and deep cupping and wide exposure of laminae, vision $6 / 6$, fields to $3 / 1000$ practically full, and no history of attacks suggesting raised tension-was to link up in theory views still somewhat at variance on the patho$g \in$ nesis of a condition not in itself likely to benefit from measures directed against raised intra-ocular pressure. In fact I go so far as to say that many needless and useless operations are to-day being done on these cases from the mistaken idea that at some time or other they have had, (though this has never been demonstrated in a given case) or will certainly have, raised tension that will do damage.

I avoid the term cavernous atrophy as if it were an adequate explanation of what may concern the lamina mainly or only.

There may or may not be an accompanying cavernous atrophy but the essential feature is a yielding of the fibrous tissue of the lamina.

Now why should this yield? Let us think again of the tennis ball. If we thin down a disc on its surface sufficiently we get a small blister-as in a motor tyre. In exactly the same way the only factor required to produce a recession of the lamina, pressure before and behind remaining the same, is a thinning or weakening of its fibres.

The possibility and probability of this happening is at once evident when one realizes that the lamina has a blood supply of its own.

This has.been demonstrated by Lagrange and Beauvieux in a paper at the meeting of the French Ophthalmological Society in 1924, which I had the pleasure of hearing. Referring to previous work they pointed out that it was impossible to inject the lamina post-mortem from the central artery of the retina. On so injecting, the parts of the nerve behind and in front of the lamina took the fluid. But on injecting the ophthalmic artery the lamina also is injected via the circle of Haller (or Zinn).

The sections show thus the absolute independence of the central artery and its non-participation in the irrigation of the lamina which depends, we repeat it, exclusively on the arterial circle of Haller. ${ }^{1}$

This circulation too seems to be terminal in character and no anastomosis with that of the central artery occurs.

The nutrition then of the fibrous lamina would be gravely compromised by any interference with its blood-supply coming from this circle; perhaps too that of the nerve elements traversing it, though, owing to the thinness of the lamina, it is conceivable that the supply from the central artery before and behind might be sufficient for these. I need not strain anatomical points however. The late Col. Elliot's paper should be consulted. ${ }^{2}$ 
Now one has only to suppose a localized narrowing of the circling vessels to give the exact condition required for a weakening of the lamina.

To demonstrate this may be impossible, but the subjects of this recession are usually past middle age and arterio-sclerosis can be demonstrated elsewhere.

The sclerosis may also involve the arterial supply to the nerve elements and so give a veritable optic atrophy. This may or may not be of cavernous type.

The essential thing to bear in mind is that such recession and even such atrophy may occur independent of any rise in intraocular pressure, and the additional factor of raised pressure is no added difficulty as Mr. Pickard supposes, when he says "It is difficult to see how it (cavernous atrophy) can arise in cases of increased tension." Lagrange's paper shows how it may and does.

We may then envisage three conditions that may combine or for a time at least remain separate and all three may occur independently of increased intra-ocular tension $(a)$ primary atrophy of the nerve elements $(b)$ cavernous atrophy involving both nerve and supporting tissue $(c)$ atrophic recession of the lamina.

No conception of retraction is needed to explain (c) nor has retracting tissue ever been demonstrated in the nerve in these cases; on the contrary the changes have been of the nature of atrophy. In this event the picture is a combined one of $(b)$ and (c), i.e., with involvement of the nerve elements.

The term "atrophic recession" seems then to be adequate to express a condition occasionally met with, of cupping without involvement of the nerve elements and without begging an increase of pressure that cannot be demonstrated. Such a recession is in our clinical experience likely to be accompanied by an atrophy first of the glial elements in front of the lamina, so leading to a wider exposure. In Elliot's view this is an effect of pressure (loc. cit. p. 312) but in the following page he agrees with Fuchs that the problem "is not the simple one of the physical effects of pressure alone."

What then may be the other factor of importance? May it not be the blood supply of the glia (central artery) and of the lamina (circle of Haller)?

It is because sufficient emphasis has not been laid on this that I have ventured to correlate these few ideas.

\section{REFERENCES}

1. Trans. of the French Soc., p. 465, 1924.

2. "On the yielding of the optic nerve head in glaucoma." Brit. Jl. of Ophthal., p. 307, 1921

3. Brit. Jl. of Ophthal., p. 332, 1931. 